\title{
Macroscopic Conductivity of Uniaxially Compacted, Sintered Balloon Aggregates ${ }^{*}$
}

\author{
Isao TAGUCHI $^{* *}$ and Michio KURASHIGE ${ }^{* * *}$ \\ ** Graduate School of Engineering, Iwate University, \\ Ueda 4-3-5, Morioka-shi, Iwate 020-8551, Japan. \\ E-mail: isao_taguchi@pref.aomori.lg.jp \\ *** Department of Mechanical Engineering, Iwate University, \\ Ueda 4-3-5, Morioka-shi, Iwate 020-8551, Japan.
}

\begin{abstract}
Balloon compacts attract a great attention as a new class of lightweight materials. In the present paper, macroscopic thermal conductivity of uniaxially compacted and sintered balloons is evaluated for various compaction degrees and balloon's inner-outer diameter ratios. The compaction is modeled by making the balloons overlapped without balloon flattening, and the sintering by adding some appropriate amount of mass around overlapping necks; the whole mass is conserved in both of the modelings. The macroscopic conductivities are estimated using Kirchhoff's current law. The results are expressed in terms of the two microgeometrical parameters. It is found that the uniaxial compaction complicatedly affects the macroscopic conductivities and strength of induced anisotropy of the compacts.
\end{abstract}

Key words: Conductivity, Simulation, Balloon, Compaction, Sintering, Random Packing

\section{Introduction}

Foams or cellular structure materials represent a new class of functional materials that have many combined advantages for many applications ${ }^{(1),(2)}$, including impact energy absorption systems, sound absorbers, compact heat exchangers, electrical and thermal insulators, and electromagnetic wave shields. In addition, highly porous materials attract increasing attention as lightweight materials in various fields, especially in such industries as aerospace, aircraft and automotive, which strongly demand mass reduction in conjunction with strength, stability or stiffness improvements.

Distinctions can be drawn between different types of porous materials, based on their cell structures. The most significant classification is that between open-cell and closed-cell structures. An obvious difference is that open-cell foams are permeable to fluid while closed cell ones are not.

Hollow spheres ${ }^{(3),(4)}$, sometimes called microballoons, have commonly been used in the fabrication of syntactic foams, which are closed-cell foams and used as core materials in sandwich composites. In recent years, bonded compacts of balloons made of metal, ceramics, inorganic glass or carbon have been fabricated ${ }^{(5)-(8)}$. Since the materials fabricated by bonding the balloons contain both types of connected (open) and closed pores, these structures have a significant potential for multifunctional applications.

Mechanical properties of hollow-sphere foams, although they are regularly packed, i.e., simple cubic, body-centered cubic and face-centered cubic, have been evaluated by the finite element analysis of a unit cell with periodic boundary conditions ${ }^{(9)-(11)}$ and compared with those for the open-cell and closed-cell foams. It has been found that the face-centered cubic 
packing gives the highest values of moduli and strength.

Bonded particle aggregates are usually fabricated by dumping particles into a container and in some cases by tapping it to obtain denser packings, leading to "random" structures. Furthermore, uniaxial or isostatic compaction of particles is a typical industrial route in their fabrication process. The combined heating and pressurization makes the particle contact areas spread and densifies the compacts ${ }^{(7)-(9)}$. This is also the case for the balloon compacts.

Thus, we need to take into account the randomness of balloon structures and the effect of pressurization and sintering. It should be noted that the randomness hinders us from using the finite element analysis of such a unit cell as in the regular stackings; we have to resort to another method.

Lightness is a common motivation for using porous materials. Thermal conductivity of such lightweight materials may also be of critical importance. Kurashige et al. ${ }^{(12)}$ proposed a simulation method for macroscopic thermal conductivities of randomly packed solid particle aggregates. It was shown that their simulation method is much better than other methods such as the self-consistent models ${ }^{(13),(14)}$. Taguchi and Kurashige ${ }^{(15)}$ used this simulation method $^{(16)}$ to predict thermal conductivity of sintered balloon aggregates. However, these simulations ${ }^{(12),(15)}$ did not take into account effects of the compaction on the thermal properties.

In the present paper, we investigate the macroscopic thermal conductivity of uniaxially pressed balloon compacts for various compaction degrees and inner-outer diameter ratios of the balloons; it is noted that conductions of heat and electrical charge are equivalent from the mathematical point of view. We take into account an effect of the uniaxial compaction in the fabrication process of compacts on the macroscopic thermal property. We use the same simulation method as in Ref. (15) and focus on the geometrical modeling of compaction and sintering complying with the mass conservation.

\section{Geometrical Model of Compacted Balloon Aggregate}

\subsection{Modeling of balloon aggregate}

In order to construct a geometrical packing model for uniaxially pressed balloon compacts in a computer, we carry out a random packing simulation. Various methods of constructing the model for random packing are available. Among the methods, we use the method of rigid-sphere successive free fall in a container, because the method is one of the simplest ones and, in addition, is appropriate to construct an adequate microgeometry of the balloon aggregate. We sequentially throw identical balloons with outer diameter $D$ from random positions into a virtual box. To simplify the algorithm, we assume that a falling particle is subjected to only gravity and forces from resting particles when collided with them. Thus, the motion equation of the particle can be expressed as follows:

$$
c \frac{d \boldsymbol{x}}{d t}=\sum k \Delta \boldsymbol{d}-m g \boldsymbol{k}
$$

where $\boldsymbol{x}$ is the position vector of a falling balloon's center, $\boldsymbol{k}$ is the unit vector along the $z$ axis and $\Delta \boldsymbol{d}$ is the overlapping depth vector of the balloon in collision with another still one. Summation notation $\sum$ is over the number of all balloons in contact with the one under consideration. Notations $c, k, m$ and $g$ are the viscosity constant, the spring constant between two balloons in collision, the mass of each balloon and the gravitational acceleration, respectively.

We use the Runge-Kutta-Gill method to solve the three coupled differential equations. In running our program, we use the following non-dimensional numerical values with balloon's outer diameter $D$ as a reference length: time increment $\Delta T=\Delta t /(\mathrm{mg} / \mathrm{cD})=1.0 \times 10^{-3}$, spring constant $K=D k /(m g)=1.0 \times 10^{3}$ and small parameter $\varepsilon=1.0 \times 10^{-7}$; the last 
parameter will be used to judge if the sphere already rests or not.

Consider a virtual box of $0 \leq x \leq B, 0 \leq y \leq B, 0 \leq z \leq H(B<H)$. We do not stop introducing balloons until the region $0 \leq x \leq B, 0 \leq y \leq B, 0 \leq z \leq 1.5 B$ is completely filled with balloons. We continue the balloon introduction up to $z=1.5 B$ because we need the margin for compaction. We adopt box sizes $B / D=16$ and five runs are made with five different sets of pseudorandom numbers. We use the periodic boundary conditions and randomly displace the floor up and down in order to avoid packing regularity on its walls and floor, respectively. The pseudorandom numbers for the initial positions and floor ups and downs are generated by algorithm of Whichmann and Hill ${ }^{(16)}$

\subsection{Modeling of compaction}

Several mechanisms can be involved in powder compaction processes ${ }^{(17)-(20)}$ : rearrangement and densification of particles, corruption of large voids and plastic deformation of particles. All these mechanisms are connected with each other and affected by friction between particles, adhesion of particles and so on; it is difficult to take into account all of them and their connection as well as various affecting factors. Therefore, we will not simulate all these processes. Furthermore, we need not know the whole process; we need only the microgeometry resulting from random packing and compaction. To describe the compacted geometry, we introduce a new scaled coordinate of each sphere center as follows:

$$
Z_{i}=(1-\beta) z_{i}
$$

where $z_{i}$ and $Z_{i}$ are the $z$ coordinate of $i$-th balloon center before and after compaction, respectively, and $\beta$ is a coefficient describing degree of the compaction, varying from 0.0 to 0.2 with 0.01 increment; $\beta=0.0$ means no compaction. It must be noted that each balloon remains spherical in spite of the center coordinate scaling, resulting to overlapping.

We extract a cubic region $B \times B \times B$ from a scaled region of the original $B \times B \times 1.5 B$. We obtain $5 \times 21$ samples in total, for each of which a list of the center coordinates of all resting particles is prepared. These lists will be used to obtain the macroscopic conductivity of pressure sintered balloons later on.

\subsection{Characteristics of compacted aggregate}

Figure 1 shows the geometries constructed by balloon packing with magnified surfaces of the aggregate in the circles. Figures $1 \mathrm{a}$ and $1 \mathrm{~b}$ show the cases of no compaction $(\beta=0.0)$ and considerable compaction ( $\beta=0.2$ ) for Sample 1, respectively. Scrutinizing the figure, we can see the displaced balloons in Fig. 1b corresponding to the original ones in Fig. 1a as shown by some arrows and many new balloons coming into the box from the upper margin in Fig. 1b.

Characteristics of these compacted aggregate are summarized as follows:

The aggregate of $\beta=0.0$ is composed of 4597 spheres, while the one of $\beta=0.2$ contains 5702 spheres. The averaged porosity of the random packings constructed by the above method before the compaction is approximately $41.7 \%$. Porosities decrease with increasing densification coefficient. For $\beta=0.2$, the averaged porosity is approximately $27.1 \%$.

We examine coordination numbers, which is defined as the average number of contact points of a particle with neighboring ones. For the compacted aggregate, heat or electric current is conducted through the necks created by the compaction, so that it is important to estimate the coordination number to know conductivities of the balloon aggregate. The mean coordination number over all balloons increase with increasing compaction coefficient, because the compaction creates new contact points; in the case of no compaction, the mean coordination number is 6.019 while that is 6.876 for $\beta=0.2$. 
A "branch" is defined as a segment connecting the centers of balloons in contact; the balloons are judged to be in contact if length of the branch is equal to or smaller than the outer balloon diameter $D$ and not in contact if larger. We examine azimuthal and zenithal distributions of orientations of the branches; the azimuthal angle is the one measured from the $x$ axis in the $x-y$ plane, while the zenithal angle is measured from the $z$-(vertical) axis. It is found from the distribution curves obtained but not shown here in the no-compaction case that the peak in the zenithal distributions appears around $50.1^{\circ}$ from the vertical line, while the azimuthal distributions are uniform in the horizontal plane. The peak angle of the zenithal distributions is $\theta=57.3^{\circ}$ for the case of considerable compaction ( $\beta=0.2$ ). From this result and simple geometrical consideration, it is seen that all the branches except almost vertical or horizontal branches incline due to the axial compaction. We will discuss the effect of increase in the branch orientations on anisotropy of the macroscopic conductivity later on.

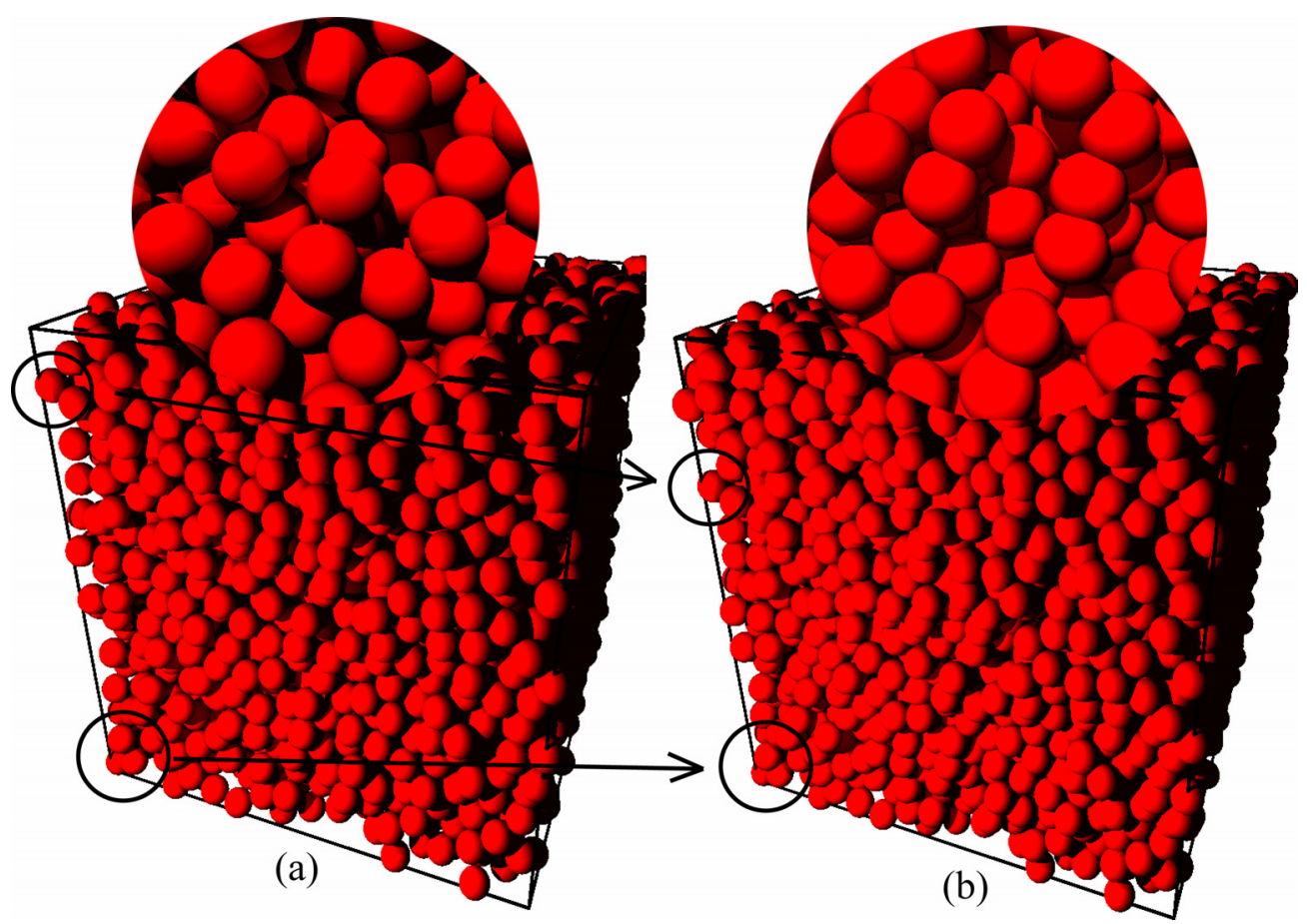

Fig.1 Constructed random packing and magnified surface of aggregate; no compaction (left) and considerable compaction (right) for Sample 1

\section{Microgeometry of Pressure Sintered Balloons}

The modeled compaction leaves overlapping of the balloons so that the overlapping results in each neck having different diameter depending on the branch orientation (and also on the compaction). In sintering the balloon aggregate, the necks grow by mass diffusion. To describe the pressure sintered geometry, we add, around the overlaps caused by the artificial reduction in balloon-center distances, some appropriate amount of mass of the same substance as that of the balloons; the added volume, as shown in Fig. 2, is the shaded portion bounded by the two overlapped balloons and a torus touching both of them.

We consider the two cases of the pressure sintered geometry separately according to whether the balloon thickness is larger than the overlapping depth or not. The first case is that this is true and is shown left in Fig. 2. In the second case shown right in the same figure, the balloon thickness is smaller than the depth. Furthermore in the latter case, a diaphragm separating the two balloons is assumed a disc whose upper edge is a cross-line of the inner 
surface of the upper balloon with the outer surface of the lower balloon and whose lower edge is its counterpart. The diaphragm thickness will be determined by this assumption. In both cases, the excess volume caused by overlapping is removed and added around the neck so as to conserve the volume (or mass).

In Fig. 2, $D$ and $d$ are the outer and inner diameter of balloons, respectively; $h$ is the overlapping depth, $h_{0}$ the diaphragm thickness and $\Theta$ the angle representing the degree of sintering.

The geometry of a pressure sintered balloon pair shown in Fig. 2 is not exactly the same as that of the real one. It is observed in the photographs ${ }^{(7),(9)}$ that the diaphragms are not flat for larger compaction. However, the flat diaphragm assumption may be accepted because the diaphragm ruggedness may not very much affect the heat conduction through the balloon pair because of little heat conduction in it, as will be proved by the distribution of entropy production rate density (see Fig. 4).

The overlapping volume and added volume per neck can be analyzed by simple geometrical consideration. For the left case of Fig. 2, the overlapping volume is as follows:

$$
\frac{4}{\pi D^{3}} V_{\text {lap }}=\frac{1}{3}\left(\frac{h}{D}\right)^{2}\left(3-\frac{h}{D}\right)
$$

The added volume is given by

$$
\begin{aligned}
\frac{4}{\pi D^{3}} V_{a d d} & =-\frac{h}{D}\left(2-\frac{h}{D}\right)\left(1-\cos \Theta-\frac{h}{D}\right) \\
& +\left(1-\frac{h}{D}\right)\left(\frac{1-\cos \Theta-h / D}{\cos \Theta}\right)^{2}\left(1+\sin ^{2} \Theta+\left(1-\cos \Theta-\frac{h}{D}-\frac{\pi}{2}+\Theta\right) \tan \Theta\right),
\end{aligned}
$$

which is also valid for the right case.

The volume conservation requires that the volume of material added around the neck should be equal to the overlapping volume. By equating Eq. (3) to Eq. (4) and solving the resulting equation by iteration, we can determine the sintering degree angle $\Theta$ for given $h / D$.

In the right case in Fig. 2, the overlapping volume is also obtained as follows: for $h \leq D-d$, this volume is given by the same equation as Eq. (3) and for $h \geq D-d$

$$
\frac{4}{\pi D^{3}} V_{l a p}=\frac{2}{3}\left\{1-\left(\frac{d}{D}\right)^{3}\right\}-\left(1-\frac{h}{D}\right)\left\{1-\left(\frac{d}{D}\right)^{2}\right\} .
$$

The volume of the diaphragm between the two balloons is

$$
\begin{aligned}
\frac{4}{\pi D^{3}} V_{\text {dia }}= & -\frac{2}{3}\left\{1-\left(\frac{d}{D}\right)^{3}\right\}+\frac{1}{3}\left(1-\frac{h}{D}\right)\left\{1+3\left(\frac{d}{D}\right)^{2}\right\} \\
& -\frac{1}{3}\left(1-\frac{h}{D}+\frac{h_{0}}{D}\right)\left\{3+\left(1-\frac{h}{D}+\frac{h_{0}}{D}\right)^{2}\right\},
\end{aligned}
$$

where

$$
\frac{h_{0}}{D}=\frac{1}{4}\left(1-\frac{d}{D}\right)\left(\frac{1+d / D}{1-h / D}\right) .
$$


Thus, the excess volume is obtained by $V_{\text {exc }}=V_{\text {lap }}-V_{\text {dia }}$. Here again, equating this excess volume to the added volume given by Eq. (4), we can determine the sintering degree angle $\Theta$ for given $h / D$ and $d / D$.
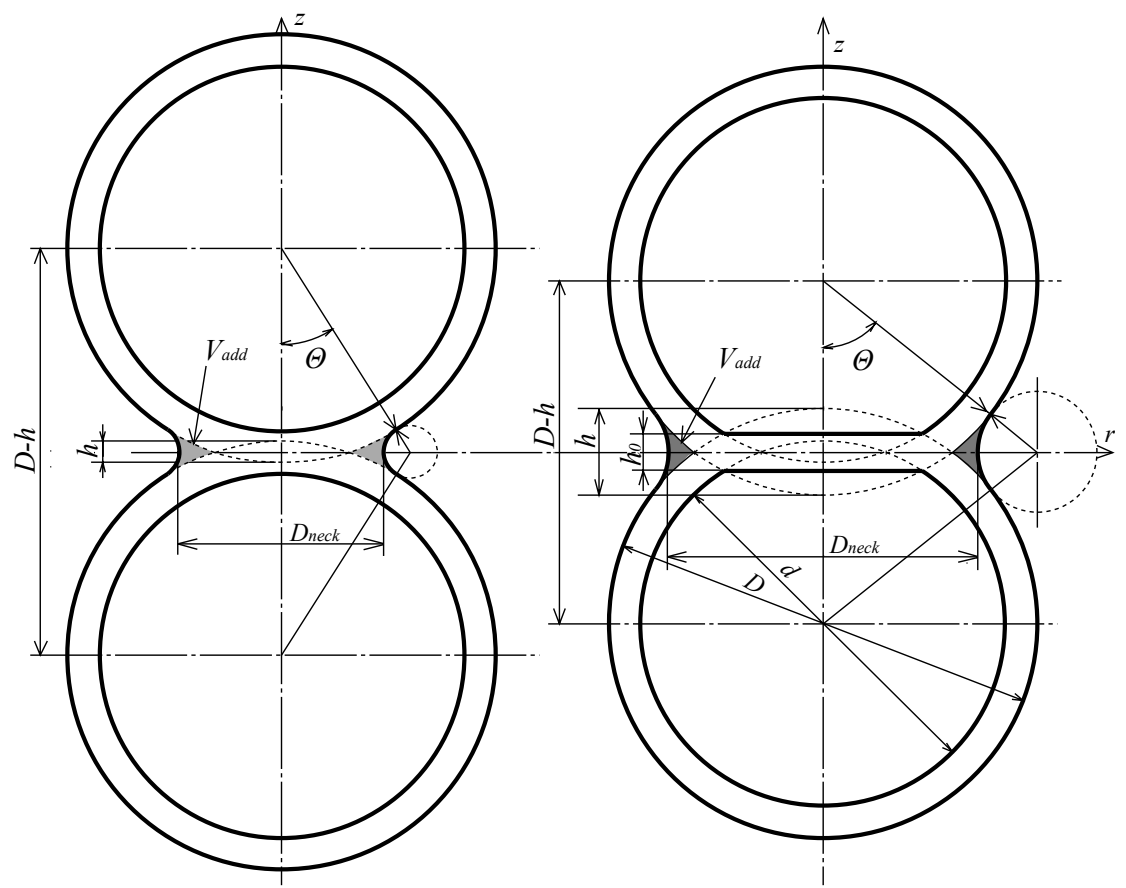

Fig. 2 Geometry model of compacted balloon pair

\section{Macroscopic Conductivity}

\subsection{Finite element analysis of conductance of balloon pair}

We calculate by the finite element analysis the conductance ratio $K / K_{0}$ of a pressure sintered balloon pair extracted from the aggregate, shown in Fig. $2 ; K_{0}$ is the axial conductance of a cylindrical solid bar with the same diameter and length as the outer balloon diameter, while $K$ the counterpart of the compacted sintered pair of balloons.

In the analysis, neither heat conduction nor convection in the vacant space of the sintered balloons and no radiation into the space are assumed, but only the heat conduction through the solid matrix of the aggregate is taken into account; so that the surfaces of the pair are insulated, that is, $\partial T / \partial n=0$ on these surfaces, where $T$ and $n$ are the temperature and the outward unit normal to them, respectively. For the sake of simplicity, the boundary conditions at both ends of the pair are assumed to be given by $T= \pm \Delta T_{0} / 2$, correspondingly. For the finite element analysis, an arbitrary cross section through the $z$ axis is divided into finite triangles, leading to triangular ring elements because of axisymmetric geometry.

The conductance ratios $K / K_{0}$ of the sintered balloon pair versus the overlapping depth $h / D$ are given in Fig. 3 for various inner-outer diameter ratios of balloons $d / D$ ranging from 0.90 to 0.95 with their increment being 0.01 .

For $\beta=0.0$, the conductance is zero for all diameter ratio $d / D$ because of the point contact. It rapidly increases with overlapping depth $h / D$ and almost linearly increases after $h / D=0.1$. The larger $d / D$, the larger the conductance. We will use these conductance ratios to evaluate the macroscopic conductivity of the aggregate in the following sub-sections. 


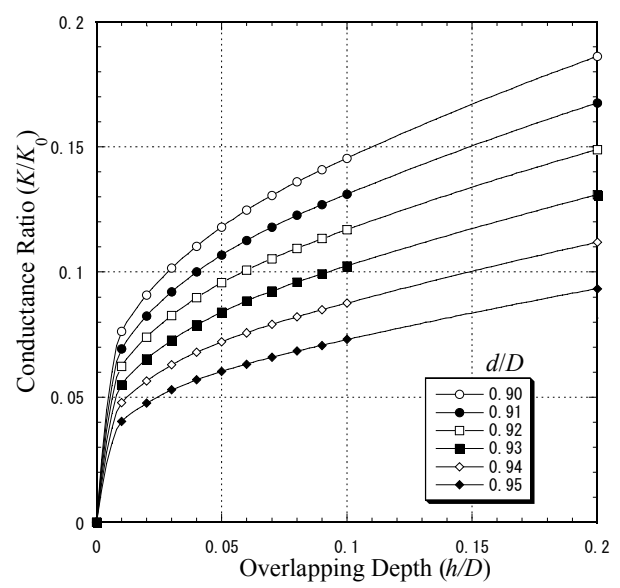

Fig. 3 Conductance ratios versus overlapping depth

\subsection{Random network of resistors}

In order to evaluate the macroscopic conductivity of the uniaxially pressed and sintered balloon compact, we will replace the aggregate by a "random network" of resistors with the conductance estimated in the above.

The replacement might be acceptable if the resistance against heat flow through the resistor concentrates around its neck. This concentrated resistance around the neck would account for the overall resistance of the resistors and consequently of the sintered aggregate composed of them. To confirm the resistance concentration, we calculate entropy production rate density within the balloon pair from the above finite element analysis. The results are shown in Fig. 4 for the cases of an almost non-compacted balloon $(\beta=0.01)$ and a well compacted balloon ( $\beta=0.20$ ) for $d / D=0.92$. It is seen from the figure that the density is highly concentrated around the corner of the neck (as depicted by the red), especially for the former case. This argument implies that the balloon wall portion which is far from the neck or sintered portion does not very much contribute to the resistance against the heat flow through the resistor. With the latter case, it should be added that, although some entropy is generated even in the wall portion far from the neck or junction as depicted by the light blue, the context just above holds because that portion has the larger cross-sectional area perpendicular to the heat flow.
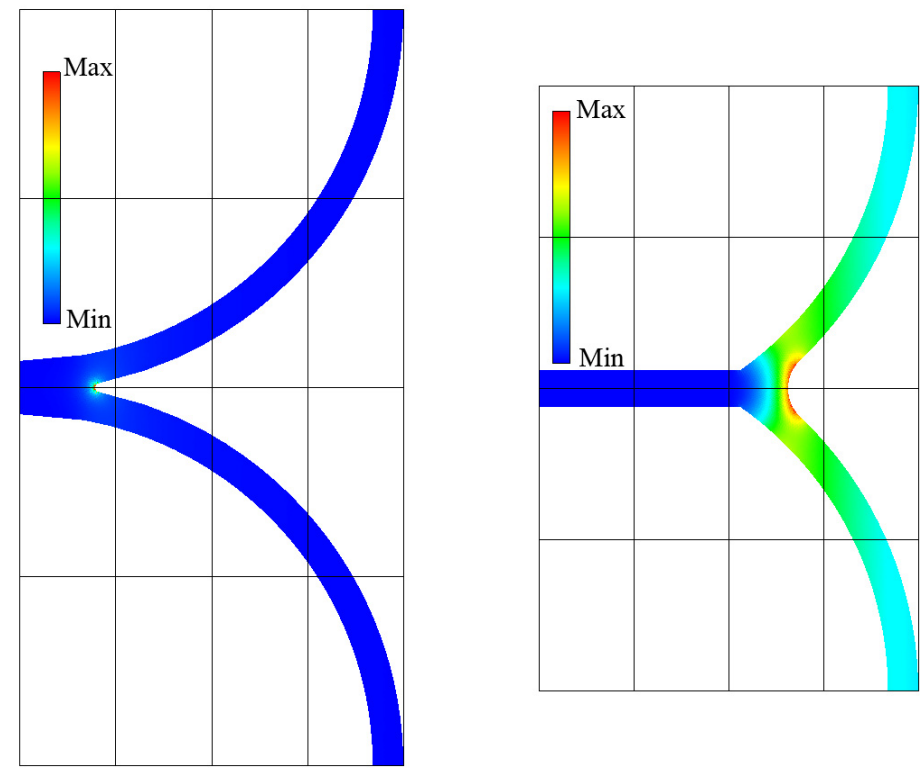

Fig. 4 Distribution of entropy production rate density 
The conductance, i.e., reciprocal of the overall resistance, of the resistors varies from resistor to resistor. To know the conductance of any of the resistors in the network, we express the relation, already determined as discrete data, between the conductance and the overlapping depth in the following form:

$$
\frac{K}{K_{0}}=C_{0}\left(\frac{h}{D}\right)^{C_{1}}+C_{2}\left(\frac{h}{D}\right)^{C_{3}}
$$

where $C_{0}$ and $C_{2}$ are the constants to be determined by the least square method after the exponents $C_{1}$ and $C_{3}$ of $(h / D)$ are fixed by the trial and error method. By calculating all branch lengths in the random network, we obtain depth $h / D$ for each resistor. From the regression curve in Eq. (8), we can obtain the required resistance of any resistor in analyzing the network.

We assign specific temperatures to the nodes (or resistors' ends, which are originally situated at the balloons' centers) existing within the outermost layers of thickness $D / 2$ in a cubic box webbed by the resistors; the values of these assigned temperatures are determined so as to be proportional to the corresponding values of coordinate in the macroscopic conduction direction, with the temperatures being zero and a given $T_{0}$ at the two opposite surfaces of the cube. The other four surfaces are assumed to be insulated. Thus, the Kirchhoff current law determines temperatures at all nodes.

Consider a central cross section of the box orthogonal to the direction of macroscopic heat flow and denote the total number of resistors crossed by this cross-section as $N$. The total heat flow rate $Q_{\text {total }}$ can be estimated by

$$
Q_{\text {total }}=\sum_{i=1}^{N} K_{i} \Delta T_{i}
$$

where $K_{i}$ is the conductance of the $i$-th resistor given by Eq. (8) and $\Delta T_{i}$ denotes the temperature difference between both the $i$-th resistor's nodes. The macroscopic conductivity is given by dividing the total flow rate by the temperature difference $T_{0}$ between those at the top and bottom of the box.

\section{Results and Discussions}

First of all, let us mention the validity of the present method. In the case of simple cubic packed and sintered "solid" particles, which is an extreme case of the present simulation, we can confirm that the present results are in considerably good agreement with the existing results $^{(21)-(23)}$ (see Fig. 5). From this agreement, it could be concluded, although not fully, that the present simulation method gives good estimates of the macroscopic conductivity of sintered balloon aggregates if we exempt the particle rearrangement during the uniaxial compaction in sintering, as mentioned above. It might be said also from some pictures ${ }^{(7)}$ and assumed geometries ${ }^{(9)-(11)}$ that the geometry of compacted and sintered balloons assumed in the present analysis well describes the reality.

The macroscopic conductivities in the $x$ and $z$ directions of the pressure sintered balloon compacts for several ratios of inner-outer diameters for Sample 1 are shown in Figs. $6 \mathrm{a}$ and $6 \mathrm{~b}$, respectively. In these figures, the ordinate presents their macroscopic conductivity $\kappa$ normalized by that of the balloon substance $\kappa_{0}$, while the abscissa is for the porosity of the sintered aggregates; this porosity takes into account the hollow volume of all balloons as well. Here, we present only the graphs for Sample 1, although we have calculated both the conductivities for all samples. Since little difference among all the samples can be seen, we can say that the sample size of $B / D=16$ is large enough to estimate the conductivity in a 
required accuracy.

We examine how the conductivity differs according to its direction. Comparison between (a) and (b) in Fig. 6 shows that the macroscopic conductivity in the $z$ direction is larger than that in the $x$ direction by roughly $20 \%$ to $35 \%$ over the entire region of porosity. The conductivity in the $y$ direction is almost exactly equal to that in the $x$ direction, although we do not show it in the form of curves here. This reveals that the simulated packing structure is of isotropy in the $x-y$ plane and of anisotropy normal to this plane. This is the case for all $\beta$ and $d / D$.

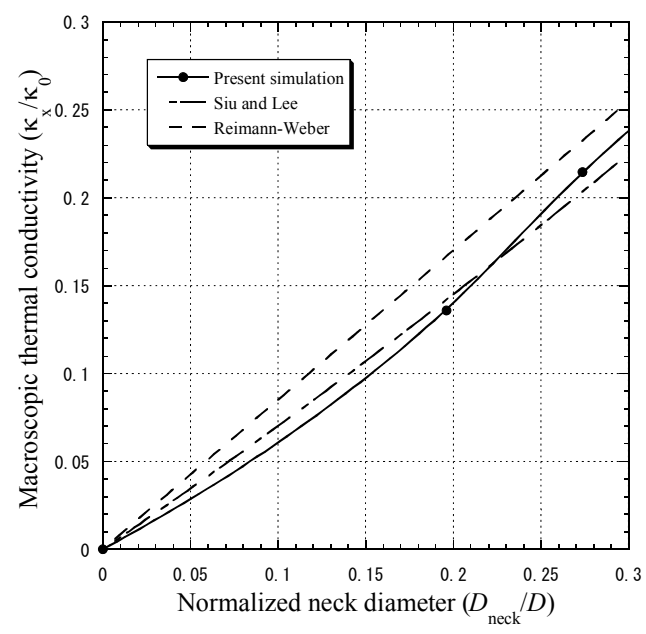

Fig. 5 Macroscopic conductivity for simple cubic packing versus normalized neck diameter
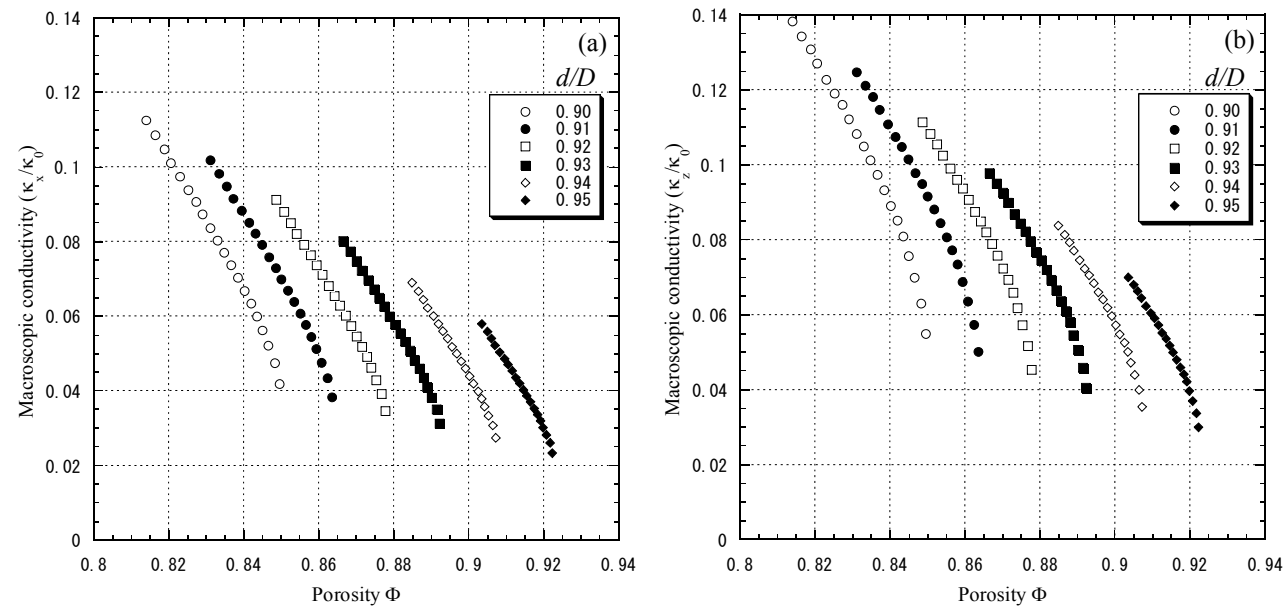

Fig. 6 Macroscopic conductivity versus porosity

Let us see how the conductivity depends on the compaction degree $\beta$ and diameter ratio $d / D$. Each marker on an individual chain of markers in both the figures corresponds to the compaction degree ranging from 0.01 to 0.20 with the interval of 0.01 ; progress toward the left on each chain makes the compaction degree higher. Each chain corresponds to different diameter ratios. For a given diameter ratio, the smaller compaction degree causes the conductivity to decrease due to the larger resistance at the thinner necks. Increases in the diameter ratio raise the conductivity curves as a whole and shift them toward lower porosity. It is noted that the macroscopic conductivities go to zero as the compaction degree tends to zero for all the diameter ratios even for a finite value of the porosity because the necks of balloons reduce to contact points.

We focus on the effect of the compaction on the degree of anisotropy. Here, we consider 
the ratio $\kappa_{z} / \kappa_{x}$. Note that $\kappa_{z} / \kappa_{x}=1$ means isotropy. This ratio is plotted against the porosity in Fig. 7 for various diameter ratios and compaction degrees. For all diameter ratios, the ratio $\kappa_{z} / \kappa_{x}$ is approximately 1.3 for $\beta=0.01$ (almost vanishing compaction). The ratios increase with increasing compaction degree up to approximately $\beta=0.04$. After passing this point, the ratios decrease with $\beta$. That is, the curve of conductivity ratio has a peak against the porosity. This peak is against our expectation that the ratio increases monotonically against the compaction degree.

To resolve this intuitive discrepancy, we can consider an effect of the microgeometry of the aggregate subjected to uniaxial compaction on the macroscopic conductivity ratio as follows: The uniaxial compaction makes the average coordination number and the inclination of the branches except almost vertical or horizontal ones larger. The more the average coordination numbers are, the larger the macroscopic conductivity is; especially, in the $z$ direction and in the early stage of compaction. This results in the increase in the conductivity ratio for the smaller compaction. The compaction-induced increase in the inclination of branches except almost vertical or horizontal ones leads to the decreasing macroscopic conductivity in the $z$ direction but to the increasing transverse conductivity. Thus, the conductivity ratio becomes less for the progressed compaction.

As a summary, the macroscopic conductivity depends not only on the microgeometry of compacted and sintered balloons, but also on the distribution of the inclination of the resistors' axis against the macroscopic conduction direction in a very complicated manner.

It is important for practical purposes to express all the conductivities in terms of the two geometrical parameters $\beta$ and $d / D$. The expressions are determined by the conventional least square method and given as follows:

$$
\begin{aligned}
& \frac{\kappa_{x}}{\kappa_{0}}=\left(4.17 \times 10^{-1}+4.72 \beta-2.3 \beta^{2}\right)\left(\frac{d}{D}\right)+\left(-4.14 \times 10^{-1}-4.76 \beta+2.3 \beta^{2}\right)\left(\frac{d}{D}\right)^{2} \\
& \frac{\kappa_{z}}{\kappa_{0}}=\left(5.59 \times 10^{-1}+6.95 \beta-9.5 \beta^{2}\right)\left(\frac{d}{D}\right)+\left(-5.56 \times 10^{-1}-7.02 \beta+9.7 \beta^{2}\right)\left(\frac{d}{D}\right)^{2}
\end{aligned}
$$

These equations describe the simulation results for $0.01 \leq \beta \leq 0.20$ and $0.90 \leq d / D \leq 0.95$ to within $1.5 \%$ in all cases.

Because of many difficulties mentioned above and as a first step of research, we have adopted the proportional reduction method for better understanding of the effect of the compaction on the macroscopic conductivity. Instead of the whole process simulation, some way of geometrical description of the particle rearrangement might be better but has not yet been found.

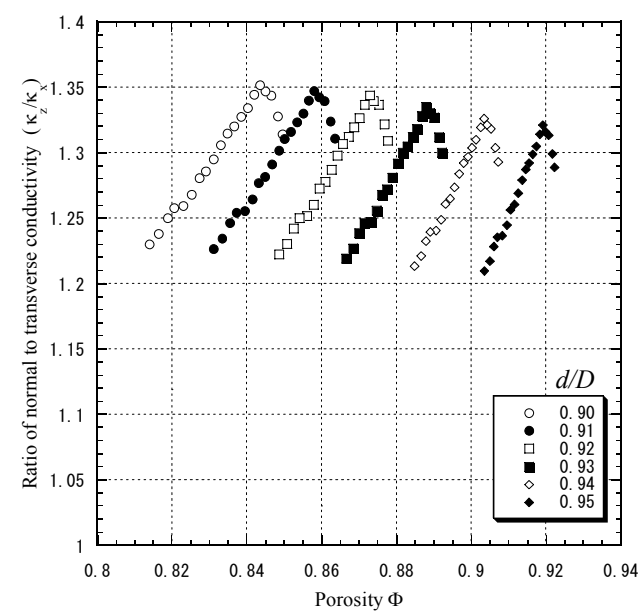

Fig. 7 Ratio of anisotropy versus porosity 
For the better design of compacts, finally, we examine a specific macroscopic conductivity defined as the macroscopic conductivity divided by effective density of the sintered aggregate, i.e., $1-\Phi$. Figure 8 shows dependence of the specific macroscopic conductivity on the porosity for various diameter ratios; (a) and (b) in this figure correspond to conductivities in the directions $x$ and $z$.

It is interesting to compare (a) and (b) in this figure with the counterparts in Fig. 6. All the curves as a whole in Fig. 8 move upwards more for the larger diameter ratio. It is because the effective density decreases as the diameter ratio increases and the degree of sintering declines; that is, the density is lower for the thinner balloon wall and for the less sintered balloon aggregate.

In Ref. (15) for the case of no compaction, the specific macroscopic conductivities against the porosity have a peak. The necks grow around contact points of the balloons by mass diffusion, soldering or brazing. To model this growing, some volume is added, leading to increase in the cross-sectional area of necks and more rapid increase in the specific density. The former increase causes sharp rise in the conductivity for the thinner neck but contributes less to the conductivity rise for the thicker neck, while the latter increase is more rapid with the thickness of the neck. This is the reason why the peak appears. It should be mentioned that the simple volume addition never violates the mass conservation, because the similarity in geometry would produce the same conductivity.

In contrast to the case of Ref. (15), there appears no peak in the present case. For the pressure sintering, the macroscopic conductivity increases with the compaction and sintering without volume addition (no increase in the specific density). Therefore, the specific macroscopic conductivities against the porosity do not have a peak.
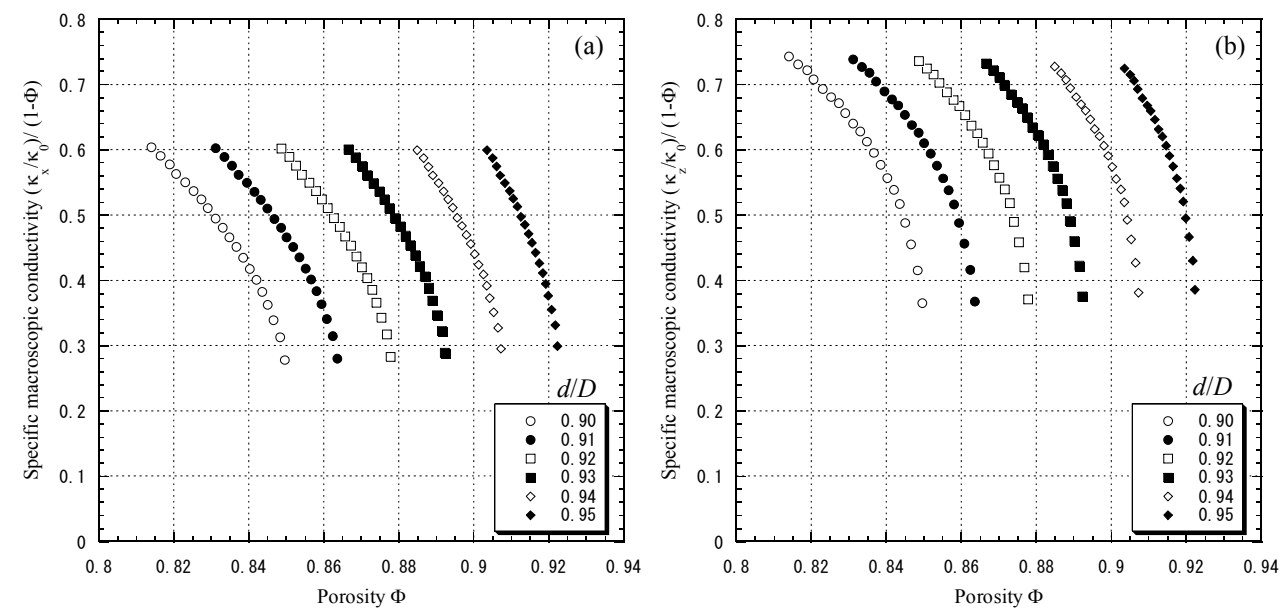

Fig. 8 Specific macroscopic conductivity versus porosity

\section{Conclusions}

Balloon compacts have a great potential as a new class of lightweight materials. We have numerically investigated the macroscopic conductivity for uniaxially pressured and sintered balloon compacts. We have confirmed that the macroscopic conductivities are of transverse isotropy. We have expressed the macroscopic conductivities in terms of the two geometrical parameters. It is found that the uniaxial compaction complicatedly affect the degree of anisotropy in the macroscopic conductivity. Furthermore, we have evaluated the specific (per weight) macroscopic conductivity and discussed its behavior in terms of the microgeometry of the balloon compacts.

\section{References}

(1) Ashby, M. F., Evans, T., Fleck, N. A., Gibson, L. J., Hutchinson, J. W. and Wadley, H. N. G., Metal Foams: A Design Guide, Butterworth-Heinemann, (2000). 
(2) Gibson, L.J. and Ashby, M.F., Cellular Solids: Structure and Properties, Second Edition, (1997), Cambridge University Press, Cambridge.

(3) Torobin, L.B., Metal microspheres, "Filamented Hollow Metal Microspheres and Articles Produced Therefrom", United States Patent No. 4582534(1986).

(4) Norris, B. and Gojny, F.J., "Hollow Metal Sphere Filled Stabilized Skin Structures and Method of Making", United States Patent No. 4925740(1990).

(5) Andersen, O., Wang, U., Schnerder, L., Stephani, G. and Kieback, B., Novel Metallic Hollow Sphere Structure, Advanced Engineering Materials, Vol. 2-4 (2000) pp. 192-195.

(6) Queheillalt, D.T., Sypeck D. J. and Wadley, H.N.G., Ultrasonic Characterization of Cellular Metal Structures, Materials Science and Engineering A, Vol. 323 (2002), pp. 138-147.

(7) Shinagawa, K., Yoshimura, H., Mihara, Y., Sukegawa, Y. and Murakami, K., Compressive Behavior of Iron Hollow Sphere at Elevated Temperatures (A Constitutive Equation for Pressure Sintering of Metallic Hollow Spheres I), Journal of the JSTP, Vol. 47 (2006), pp. 323-327.

(8) Shinagawa, K., Yoshimura, H., Mihara, Y., Sukegawa, Y. and Murakami, K., Experimental and Simulation of Firing of Iron Hollow Sphere Compacts (A Constitutive Equation for Pressure Sintering of Metallic Hollow Spheres II), Journal of the JSTP, Vol. 47 (2006), pp. 328-332.

(9) Sanders, W.S. and Gibson, L.J., Mechanics of Hollow Sphere Foams, Materials Science and Engineering A, Vol. 347 (2003), pp. 70-85.

(10) Sanders, W.S. and Gibson, L.J., Mechanics of BCC and FCC Hollow-Sphere Foams, Materials Science and Engineering A, Vol. 352 (2003), pp. 150-161.

(11) Gasser, S. et al., Uniaxial Tensile Elastic Properties of a Regular Stacking of Brazed Hollow Spheres, Scripta Materialia, Vol. 48 (2003), pp. 1617-1623.

(12) Kurashige, M., Mishima, M. and Imai, K., Simulated Effective Thermal Conductivity of Sintered, Randomly Packed Spheres and Statistical Structures of Packings, Journal of Thermal Stresses, Vol. 22 (1999), pp. 713-733.

(13) Hill, R., Self-Consistent Mechanics of Composite Materials, Journal Mech. Phys. Solids, Vol. 13 (1965), pp. 213-222.

(14) Taya, M. and Arsenault, R.J., Metal Matrix Composite, (1989), Pergamon Press.

(15) Taguchi, I. and Kurashige, M., Statistical Properties of Random Packing and Macroscopic Thermal Conductivity of Sintered Balloons: Re-examination, Transactions of the Japan Society of Mechanical Engineers, Series A, Vol. 72, No.722 (2006), pp. 1577-1584.

(16) Wichmann, B.A. and Hill, I.D., An Efficient and Portable Pseudo-Random Number Generator, Royal Statistical Society, Algorithm as 183 (1982), pp. 188-190.

(17) Martin, C.L. and Bouvard D., Isostatic Compaction of Bimodal Powder Mixtures and Composites, International Journal of Mechanical Sciences, Vol. 46 (2004), pp. 907-927.

(18) Martin, C.L. and Bouvard D., Study of the Cold Compaction of Composite Powders by the Discrete Element Method, Acta Materialia, Vol. 51 (2003), pp. 373-386.

(19) Kim, K.T., Lee, S.C. and Ryu, H.S., Densification Behavior of Aluminum Alloy Powder Mixed with Zirconia Powder Inclusion Under Cold Compaction, Materials Science and Engineering A, Vol. 340 (2003), pp. 41-48.

(20) Redanz, P. and Fleck, N.A., The Compaction of a Random Distribution of Metal Cylinders by the Discrete Element Method, Acta Materialia, Vol. 49 (2001), pp. 4325-4335.

(21) Gusarov, A.V., Laoui, T., Froyen, L. and Titov, V.I., Contact Thermal Conductivity of a Powder Bed in Selective Laser Sintering, International Journal of Heat and Mass Transfer, Vol. 46 (2003), pp. 1103-1109.

(22) Siu, W.W.M. and Lee, S.H.-K., Effective Conductivity Computation of a Packed Bed Using Constriction Resistance and Contact Angle Effects, International Journal of Heat and Mass Transfer, Vol.43 (2000), pp. 3917-3924.

(23) Luikov, A.V., Heat and Mass Transfer, Handbook, (1971), Energia, Moscow. 